\title{
The Kazhdan Property of the Mapping Class Group of Closed Surfaces and the First Cohomology Group of Its Cofinite Subgroups
}

Feraydoun Taherkhani

\section{CONTENTS}

1. Introduction

2. Constructing Examples for $g=2$

3. Constructing Examples for $g=3$

4. Constructing $\Phi_{\mathrm{g}, \mathrm{Q}}$

5. Computing a Presentation for $\mathrm{H}$

6. More Examples for $g=2$

7. Final Remarks

Electronic Availability

References
We show that the mapping class group of a closed surface of genus 2 does not satisfy the Kazhdan property by constructing subgroups of finite index having a nonvanishing first cohomology group. We also construct some subgroups of finite index in the mapping class group of a genus 3 surface and calculate their first cohomology groups, which all turn out to be trivial. Most of the calculations have been carried out by computer using GAP.

\section{INTRODUCTION}

Let $\mathcal{S}_{g}$ be a closed surface of genus $g$, with fundamental group $\pi_{g}=\pi_{1}\left(\mathcal{S}_{g}\right)$ and corresponding mapping class group $\mathcal{M}_{g}$; recall that $\mathcal{M}_{g}$ is the group of the isotopy classes of orientation preserving diffeomorphisms of $\mathcal{S}_{g}$. The connection between $\mathcal{M}_{g}$ and combinatorial group theory was established by Nielsen $[1927 ; 1929 ; 1931] . \mathcal{M}_{g}$ has index two in the extended mapping class group $\mathcal{M}_{g}^{*}$ (the group of isotopy classes of not necessarily orientation preserving diffeomorphisms), which from is isomorphic to $\operatorname{Out}\left(\pi_{g}\right)$, the outer automorphism group of $\pi_{g}$. Hence $\mathcal{M}_{g}$ can be identified with a subgroup of index two in $\operatorname{Out}\left(\pi_{g}\right)$, which we denote by $\mathrm{Out}^{+}\left(\pi_{g}\right)$. The group $\mathcal{M}_{g}$ is generated by Dehn twists around simple closed curves [Dehn 1938]. Unaware of Dehn's result, Lickorish [1964] found a generating set of $3 g-1$ elements. The minimal number of Dehn twists generating $\mathcal{M}_{g}$ is $2 g+1$ and was determined by Humphries [1979]. The Dehn twists around the simple closed curves $\alpha_{1}, \beta_{1}, \alpha_{2}, \beta_{2}, \ldots, \alpha_{g}, \beta_{g}, \delta$ of Figure 1 will be our choice for a generating set and we will denote them by $D_{\alpha_{1}}, D_{\beta_{1}}, D_{\alpha_{2}}, D_{\beta_{2}}, \ldots, D_{\alpha_{g}}$, $D_{\beta_{g}}, D_{\delta}$. 


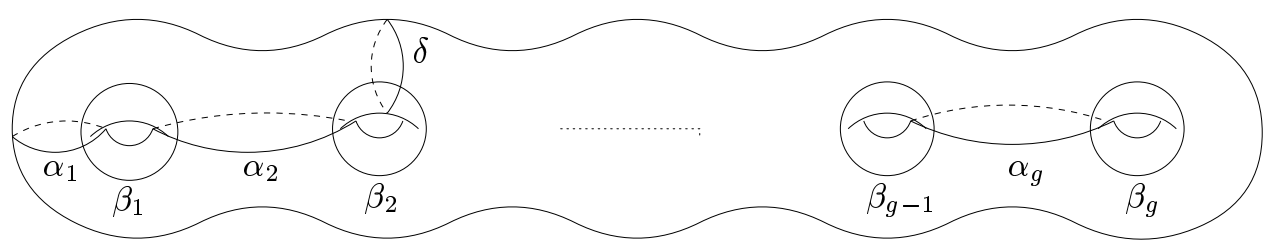

FIGURE 1. Dehn twists generating $\mathcal{M}_{g}$.

The question of the existence of a finite presentation was settled in [Birman and Hilden 1971] for the case of $g=2$ and in [McCool 1975] for $g \geq 3$. A simple presentation was determined (and later corrected) by Wajnryb [1983], based on results obtained by Hatcher and Thurston. Directly from the presentation, we can establish the well-known result that $\mathcal{M}_{g}$ is a perfect group for $g \geq 3$ [Powell 1978]:

$$
H_{1}\left(\mathcal{M}_{g}, \mathbb{Z}\right)= \begin{cases}\mathbb{Z}_{10} & \text { if } g=2 \\ 0 & \text { if } g \geq 3\end{cases}
$$

Another property of $\mathcal{M}_{g}$ is its residual finiteness, proved in [Grossman 1974]. Residual finiteness of $\mathcal{M}_{g}$ means that the intersection of all of its normal subgroups of finite index is trivial [Magnus et al. 1966, p. 116]. In the mid-eighties, the analogy between $\mathcal{M}_{g}$ and the arithmetic groups was established by Ivanov $[1987 ; 1984 ; 1986]$. For the definition of an arithmetic group we refer to [Humphreys 1980]. However for our purposes the only arithmetic group of interest would be the symplectic group over the integers, $\mathrm{Sp}_{2 g}(\mathbb{Z})$, for $g \geq 2$.

Theorem 1.1. Let $\Gamma_{g}(m)$ be the kernel of the canonical epimorphism

$$
\Phi_{g}(m): \operatorname{Sp}_{2 g}(\mathbb{Z}) \rightarrow \operatorname{Sp}_{2 g}\left(\mathbb{Z}_{m}\right) .
$$

(a) Every cofinite subgroup of $\mathrm{Sp}_{2 g}(\mathbb{Z})$ (every subgroup of finite index) contains one of the congruence subgroups $\Gamma_{g}(m)$.

(b) Every nontrivial normal subgroup in $\mathrm{Sp}_{2 g}(\mathbb{Z})$ different from the center contains a congruence subgroup and hence is of finite index.

(c) Every cofinite subgroup $U$ of $\mathrm{Sp}_{2 g}(\mathbb{Z})$ has a vanishing first cohomology group; that is, $H^{1}(U)=$ 0. Since $\mathrm{Sp}_{2 g}(\mathbb{Z})$ is finitely presented, this property is equivalent to saying that $U / U^{\prime}$ is finite.
Proof. Parts (a) and (b) are proved in [Mennicke 1965] the symplectic group and generalized in [Bass et al. 1967]. Part (c) of the theorem is a consequence of part (b) and the following proposition:

Proposition 1.2. Let $H \leq G$ be cofinite. If $\left[H: H^{\prime}\right]$ is finite, so is $\left[G: G^{\prime}\right]$.

Proof. Since $H^{\prime} \leq G^{\prime}$, therefore $H^{\prime} \leq G^{\prime} \cap H$ and consequently $G^{\prime} \cap H$ will be cofinite in $G$, but $G^{\prime} \cap$ $H \leq G^{\prime}$, which means that $G^{\prime}$ is cofinite in $G$.

To prove part (c) of the theorem, we may assume that $U$ is a normal subgroup of $G=\mathrm{Sp}_{2 g}(\mathbb{Z})$; otherwise we can pass to $\operatorname{Core}_{G}(U)=\bigcap_{g \in G} g^{-1} U g$, which is a cofinite normal subgroup in $G$ contained in $U$. Using the proposition, it suffices to prove the statement for $\operatorname{Core}_{G}(U)$. Since $U$ is a normal subgroup of $G$, therefore $U^{\prime}$ as a characteristic subgroup of $U$ will be a normal subgroup of $G$. Using part $b$ of the theorem, $U^{\prime}$ is cofinite in $G$ hence cofinite in $U$.

Let $N \unlhd \pi_{g}$ be a cofinite characteristic subgroup of $\pi_{g}$. Then the canonical map

$$
\operatorname{Aut} \pi_{g} \rightarrow \operatorname{Aut}\left(\pi_{g} / N\right)
$$

factors through the outer automorphism group, and after the restriction to $\mathcal{M}_{g}$ - recalling that $\mathcal{M}_{g} \simeq$ $\mathrm{Out}^{+}\left(\pi_{g}\right)$ - we obtain a homomorphism

$$
\Psi_{g, N}: \mathcal{M}_{g} \rightarrow \operatorname{Out}\left(\pi_{g} / N\right)
$$

whose kernel $\Lambda_{g, N}$ is a cofinite normal subgroup in $\mathcal{M}_{g}$. We call these subgroups the congruence subgroups, in analogy with arithmetic groups. N. Ivanov [1994] asks these questions about $\mathcal{M}_{g}$ :

1. Congruence Subgroup Problem for $\mathcal{M}_{g}$ : Does any cofinite subgroup $U \leq \mathcal{M}_{g}$ contain one of the $\Lambda_{g, N}$ ?

2. Does every cofinite subgroup $U$ of $\mathcal{M}_{g}$ have a vanishing first cohomology group?

3. Does $\mathcal{M}_{g}$ satisfy the Kazhdan property? 
For an introduction to the Kazhdan property (or property $T$ ) see [Kazhdan 1967; de la Harpe and Valette 1989; Lubotzky 1994]. What we need to know here is that if a group satisfies the Kazhdan property the first cohomology group of all its cofinite subgroups vanishes. Thus a negative response to the second question would answer the third question negatively as well.

Theorem 1.3. The mapping class group of a closed surface of genus $g=2$ does not have the Kazhdan property.

We will prove Theorem 1.3 by constructing examples of subgroups having a nontrivial first cohomology group.

\section{CONSTRUCTING EXAMPLES FOR $g=2$}

$\mathcal{M}_{g}$ acts on the first homology groups $H_{1}\left(\mathcal{S}_{g}, \mathbb{Z}\right)$ and $H_{1}\left(\mathcal{S}_{g}, \mathbb{Z}_{m}\right)$ for every $m \in \mathbb{Z}$. This action preserves the symplectic form and gives rise to the homomorphisms

$$
\Theta_{g}: \mathcal{M}_{g} \rightarrow \mathrm{Sp}_{2 g}(\mathbb{Z})
$$

and

$$
\Theta_{g}(m): \mathcal{M}_{g} \rightarrow \operatorname{Sp}_{2 g}\left(\mathbb{Z}_{m}\right),
$$

which are known to be surjective. Let $\mathcal{T}_{g}=\operatorname{Ker} \Theta_{g}$ be the Torelli subgroup of $\mathcal{M}_{g}$ and

$$
\mathcal{T}_{g}(m)=\operatorname{Ker} \Theta_{g}(m)
$$

the preimage of the congruence subgroup $\Gamma_{g}(m)$ in $\mathcal{M}_{g}$. In this way, we obtain a lot of cofinite normal subgroups of $\mathcal{M}_{g}$ all containing $\mathcal{T}_{g}$. In particular, in the case of $g=2$ and $m=2$ we get

$$
\Theta_{2}(2): \mathcal{M}_{2} \rightarrow \operatorname{Sp}_{4}\left(\mathbb{Z}_{2}\right) \simeq S_{6} .
$$

$\mathrm{Sp}_{4}\left(\mathbb{Z}_{2}\right)$ is isomorphic to $S_{6}$, the symmetric group on six elements, which has order 720 . Therefore $\mathcal{T}_{2}(2) \unlhd \mathcal{M}_{2}$ will be a normal subgroup of index 720 in $\mathcal{M}_{2}$. The normal subgroup $\mathcal{T}_{2}(2)$ is generated by the squares of the Dehn twists around the simple closed curves and normally generated by the square of only one of the Dehn twists, such as $a_{1}$, the first generator of $\mathcal{M}_{2}$ [Humphries 1992]. Using the Schreier-Reidemeister method [Johnson 1976; $1980 \mathrm{~b}]$, we can calculate a presentation for $\mathcal{T}_{2}(2)$ using GAP [Schönert et al. 1996]. (We have used GAP version 3 release 4.4 of April 18, 1997.) The simplest presentation we can construct, after all the possible reductions using Tietze transformations contains 14 generators and 388 relations of total length 8622 . As a subgroup, $\mathcal{T}_{2}(2)$ is generated by the 14 elements $a_{1}^{-2}, b_{1}^{-2}, a_{2}^{-2}, b_{2}^{-2}, d^{-2}, a_{1} b_{1}^{-2} a_{1}^{-1}, b_{1} a_{2}^{-2} b_{1}^{-1}$, $a_{2} b_{2}^{-2} a_{2}^{-1}, b_{2} d^{-2} b_{2}^{-1}, a_{1} b_{1} a_{2}^{-2} b_{1}^{-1} a_{1}^{-1}, a_{2} b_{2} d^{-2} b_{2}^{-1} a_{2}^{-1}$, $a_{1} b_{1} a_{2} b_{2}^{-2} a_{2}^{-1} b_{1}^{-1} a_{1}^{-1}, b_{1} a_{2} b_{2}^{-2} a_{2}^{-1} b_{1}^{-1}$, and

$$
b_{1} a_{2} b_{2} d^{-2} b_{2}^{-1} a_{2}^{-1} b_{1}^{-1} \text {. }
$$

By writing the 388 relations in additive form, we obtain a matrix of 388 rows and 14 columns that we refer to as the relation matrix of the presentation. From the presentation, we can compute $H_{1}\left(\mathcal{T}_{2}(2)\right)$ (the commutator factor group of $\mathcal{T}_{2}(2)$ ) by applying the Gaussian algorithm to this matrix to evaluate its invariant divisors. The divisors are

$$
0,0,0,0,0,0,0,0,0,2,2,2,2,4
$$

meaning that

$$
H_{1}\left(\mathcal{T}_{2}(2)\right)=9 \mathbb{Z} \oplus 4 \mathbb{Z}_{2} \oplus \mathbb{Z}_{4} .
$$

As a byproduct, we see that 14 is the lowest cardinality for a generating set of this group ( since none of the invariant divisors is 1 ). This example takes care of the genus $g=2$.

In addition, using an algorithm called LowIndexSubgroups in GAP we can calculate a complete list of the conjugacy classes of all subgroups of finite index bounded by a given number $p$. (The algorithm is efficient only for small indices. For example, $p=20$ is already a huge index for $\mathcal{M}_{2}$.) We have tabulated all the conjugacy classes of subgroups of $\mathcal{M}_{2}$ for $p=10$ together with their commutator factor group in Table 1.

\begin{tabular}{ccc} 
Index & $H$ & $H / H^{\prime}$ \\
\hline 1 & $H_{1}$ & $\mathbb{Z}_{10}$ \\
2 & $H_{2}$ & $\mathbb{Z}_{5}$ \\
3 & - & - \\
4 & - & - \\
5 & $H_{3}$ & $\mathbb{Z}_{2}$ \\
6 & $H_{4}$ & $\mathbb{Z}_{10}$ \\
6 & $H_{5}$ & $\mathbb{Z}_{80}$ \\
7 & - & - \\
8 & - & - \\
9 & - & - \\
10 & $H_{6}$ & 0 \\
10 & $H_{7}$ & $\mathbb{Z} \oplus \mathbb{Z}_{2}$
\end{tabular}

TABLE 1. Subgroups of low index in $\mathcal{M}_{2}$. 
Actually, $H_{6}$ is the commutator subgroup of $\mathcal{M}_{2}$, which is a perfect group, and $H_{7}$ corresponds to the only subgroup (up to conjugation) of order 72 in $S_{6}$. Hereby, we have found the smallest index subgroup with nontrivial first cohomology group. Using the same method, we can find a generating set, together with a presentation for $H_{7}$ :

$$
H_{7}=\left\langle b_{1}, a_{2}^{-2}, b_{2}, a_{2} b_{1} a_{1} b_{2} a_{2} d^{-1} b_{2}^{-1} b_{1}^{-1} a_{2}^{-1}\right\rangle .
$$

Since $H_{7}$ has a relatively small index and is generated by only four elements, the procedure of finding a presentation can even be done by hand. The simplest presentation we can construct for $H_{7}$ consists of 4 generators and 25 relations of total length 564 . Table 2 shows this presentation explicitly.

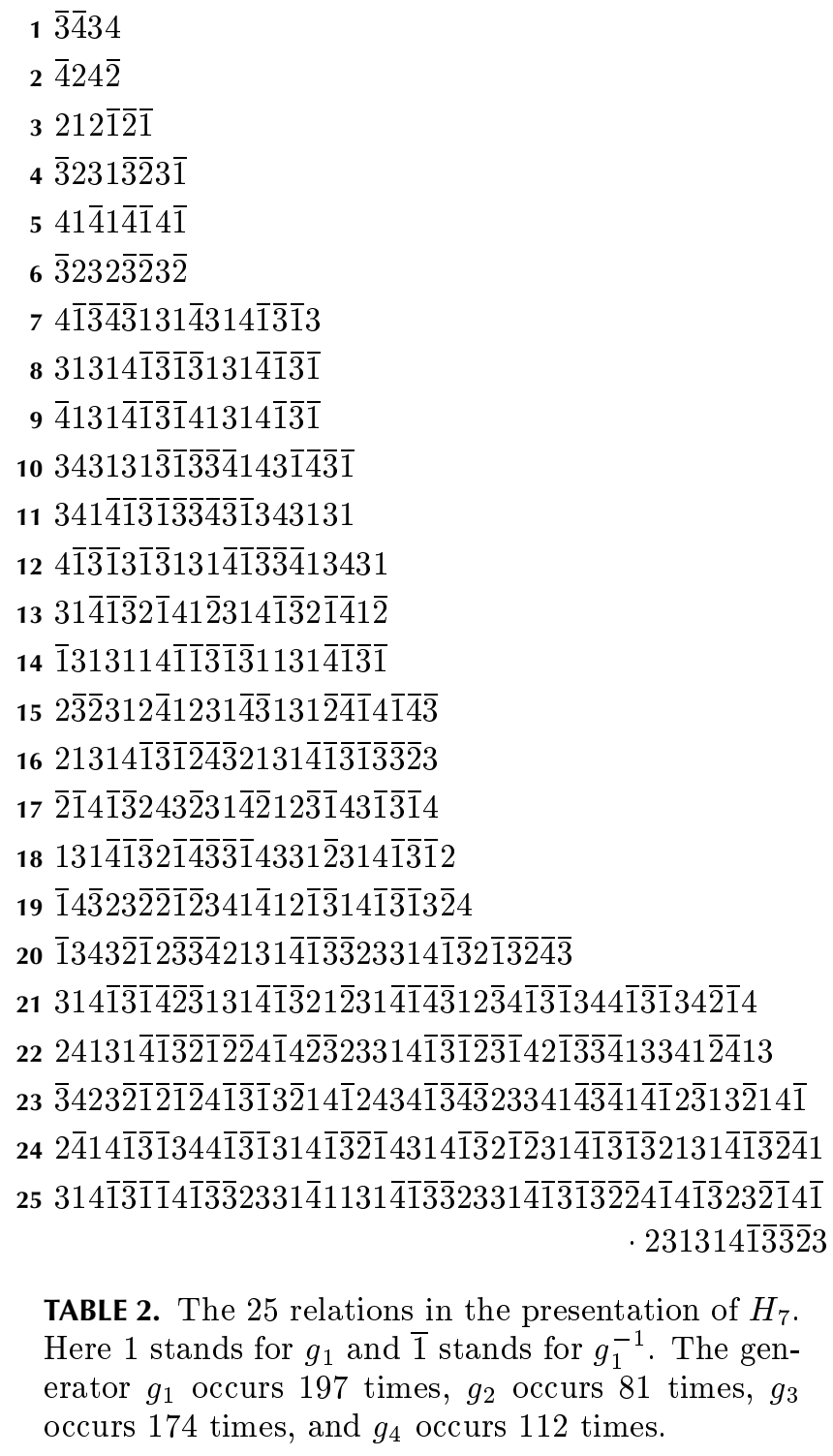

TABLE 2. The 25 relations in the presentation of $H_{7}$. Here 1 stands for $g_{1}$ and $\overline{1}$ stands for $g_{1}^{-1}$. The generator $g_{1}$ occurs 197 times, $g_{2}$ occurs 81 times, $g_{3}$ occurs 174 times, and $g_{4}$ occurs 112 times.

At the end, we calculate the commutator factor group of $H_{7}$. The invariant divisors of the relation matrix are 1, 1, 2, 0, meaning that

$$
H_{1}\left(H_{7}\right)=\mathbb{Z}_{2} \oplus \mathbb{Z},
$$

or

$$
H^{1}\left(H_{7}\right)=\mathbb{Z} .
$$

This proves Theorem 1.3.

\section{CONSTRUCTING EXAMPLES FOR $g=3$}

The result for genus $g=2$ is not very surprising, because of the exceptional status of $g=2$. There are many properties that all surfaces of $g \geq 3$ share, but a surface of $g=2$ does not [Johnson 1983]. Therefore, the interesting examples would be for surfaces of higher genera.

The case $g=3$ is not only much more difficult to handle but also quite different in nature. The first reason is the following theorem, proved in [McCarthy 1996].

Theorem 3.1. Let $\Gamma \leq \mathcal{M}_{g}$ be a subgroup of finite index containing the Torelli subgroup $\mathcal{T}_{g}$. Then $H^{1}(\Gamma)=0$.

The proof uses a result from [Johnson 1980a] and the fact that the image of $\Gamma$ in $\operatorname{Sp}_{2 g}(\mathbb{Z})$ contains some congruence subgroup. According to this theorem, if a cofinite subgroup of $\mathcal{M}_{g}$ with nontrivial first cohomology group exists, it has to be found among those that do not contain $\mathcal{T}_{g}$. The residual finiteness of $\mathcal{M}_{g}$ assures us the existence of subgroups not containing the Torelli subgroup. The main problems we encounter for the construction of these subgroups are:

1. How to find cofinite subgroups of $\mathcal{M}_{g}$ ?

2. How to check whether they contain $\mathcal{T}_{g}$ or not?

3. How to calculate the first cohomology groups of these subgroups?

Problem 1. In order to construct a whole series of cofinite subgroups of $\mathcal{M}_{g}$, we have adopted a method that was originally introduced by R. Gilman [1977] to study the automorphism groups of free groups. We have modified this method and have applied it to $\mathcal{M}_{g}$ as follows:

Let $Q$ be a finite group and $G$ a finitely presented group. Two epimorphisms $\varphi_{1}$ and $\varphi_{2}$ from $G$ onto $Q$ have the same kernel if and only if they differ by an automorphism of $Q$; that is, $\varphi_{1}=\psi \varphi_{2}$, where 
$\psi \in$ Aut $Q$. If we denote the set of all epimorphisms of $G$ onto $Q$ by $\operatorname{Epim}(G, Q)$, we have the bijection

$$
\mathcal{N}_{Q}=\{N \unlhd G: G / N \simeq Q\} \leftrightarrow \operatorname{Epim}(G, Q) / \text { Aut } Q .
$$

Now let $G=\pi_{g}$. The automorphism group of $\pi_{g}$ acts on $\mathcal{N}_{Q}$ as a permutation group. Let $k$ be the cardinality of $\mathcal{N}_{Q}$ and $S_{k}$ the symmetric group on $k$ elements. We obtain a homomorphism

$$
\Phi_{g, Q}: \text { Aut } \pi_{g} \rightarrow S_{k}
$$

The inner automorphisms act trivially on $\mathcal{N}_{Q}$. Thus $\Phi_{g, Q}$ factors through the outer automorphism group of $\pi_{g}$. After restriction to $\mathcal{M}_{g}$, we obtain a homomorphism

$$
\Phi_{g, Q}: \mathcal{M}_{g} \rightarrow S_{k}
$$

$\Sigma_{g, Q}$, the kernel of $\Phi_{g, Q}$, will be a cofinite normal subgroup of $\mathcal{M}_{g}$. In this way, we obtain a lot of different normal subgroups of $\mathcal{M}_{g}$ for different choices of $Q$.

Remark 3.2. Let $N \in \mathcal{N}_{Q}$. Then

$$
H:=\bigcap_{\varphi \in \operatorname{Aut} \pi_{g}} \varphi(N)
$$

will be a cofinite characteristic subgroup of $\pi_{g}$, and $\Lambda_{g, H} \leq \Sigma_{g, Q}$. In particular, each of the subgroups $\Sigma_{g, Q}$ contains at least one congruence subgroup.

Problem 2. Based on Johnsons' results on the Torelli subgroup [Johnson 1983], we will give a method to determine whether $\mathcal{T}_{g} \leq \Sigma_{g, Q}$ or not. This will be discussed in Section 4, when we construct some subgroups.

Problem 3. This is the most difficult part of the calculation to deal with. In order to determine $H^{1}(U)$, a presentation of $U$ has to be given. The algorithms we use to determine a presentation for a subgroup of a finitely presented group are based on a classical theorem from combinatorial group theory:

Theorem 3.3 (Schreier-Reidemeister). Let $G$ be a finitely presented group with $n$ generators and $m$ relations. In addition, let $H \leq G$ be a subgroup of finite index with $[G: H]=p$. Then $H$ is also finitely presented, and there is an algorithm to construct a presentation for $H$ on $p n-p+1$ generators and at most $m p$ relations.
There are generally two different methods to construct such a presentation, one due to Schreier and Reidemeister, and the other due to Todd and Coxeter [Johnson 1976; 1980b]. Modified versions of both algorithms have been implemented on GAP. As we see from the theorem, the complexity of the presentation increases with the index $p$. In addition, the length of the defining relations for $H$ depends on $p$ as well and can become eventually very large. (See the presentation that was constructed for $\mathcal{T}_{2}(2)$ in the previous section. For instance, the first relation is a word of length 4. Meanwhile the length of the last relation is 50.) Therefore, constructing presentations for subgroups of huge indices can become an infeasible task, (for instance $p=1000$ is already huge in the case of $\left.\mathcal{M}_{2}(2)\right)$.

In the next sections, we consider some examples for different choices of $Q$. At first, we show that abelian $Q$ 's are of no help for our purposes.

Example 3.4. $Q=\mathbb{Z}_{2}$.

Aut $\mathbb{Z}_{2}$ is trivial. Hence there are as many subgroups of index 2 in $\pi_{3}=\pi_{1}\left(\mathcal{S}_{3}\right)$ as different epimorphisms from $\pi_{3}$ onto $\mathbb{Z}_{2}$. Therefore, there are exactly $2^{6}-$ $1=63$ subgroups of index 2 in $\pi_{3}$. Consequently, we obtain the homomorphism

$$
\Phi_{2, \mathbb{Z}_{2}}: \mathcal{M}_{3} \rightarrow S_{63}
$$

The action of $\mathcal{M}_{3}$ on these subgroups coincides with the symplectic action of $\mathcal{M}_{3}$ on the subspaces of codimension 1 in $\left(\mathbb{Z}_{2}\right)^{6} \simeq H_{1}\left(\mathcal{S}_{3}, \mathbb{Z}_{2}\right)$. Therefore, $\Phi_{2, \mathbb{Z}_{2}}$ factors through $\operatorname{Sp}_{6}\left(\mathbb{Z}_{2}\right)$ and gives us a faithful permutation representation of $\mathrm{Sp}_{6}\left(\mathbb{Z}_{2}\right)$, which is also transitive (see [Huppert 1967] page 221). This implies that $\Sigma_{3, \mathbb{Z}_{2}}=\mathcal{T}_{3}(2)$ is a normal subgroup of index $\left|\operatorname{Sp}_{6}\left(\mathbb{Z}_{2}\right)\right|=1451520$ that contains $\mathcal{T}_{g}$, and consequently, $H^{1}\left(\Sigma_{3, \mathbb{Z}_{2}}\right)=0$.

Theorem 3.5. For every abelian $Q, H^{1}\left(\Sigma_{g, Q}\right)=0$.

Proof. $\mathcal{T}_{g}$ acts trivially on $H_{1}\left(\mathcal{S}_{g}, \mathbb{Z}\right)$. Since $Q$ is abelian, every $N \unlhd \pi_{g}$ with $\pi_{g} / N \simeq Q$ contains $\pi_{g}^{\prime}$. As a result, $\mathcal{T}_{g}$ acts trivially on $\left\{N: \pi_{g} / N \simeq Q\right\}$, that is, $\mathcal{T}_{g} \leq \Sigma_{g, Q}$.

In the next section, $Q$ will be always a nonabelian group, and we will describe the procedure to construct the homomorphism $\Phi_{g, Q}$ and its kernel $\Sigma_{g, Q}$. 


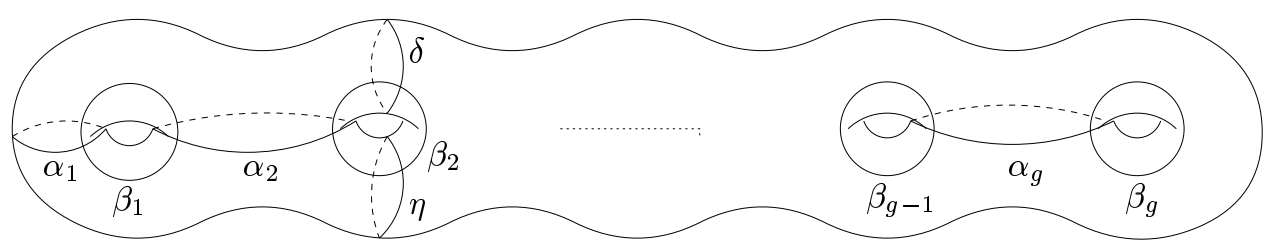

FIGURE 2. The bounding pair generating $\mathcal{T}_{g}$.

\section{CONSTRUCTING $\Phi_{\mathrm{g}, \mathrm{Q}}$}

We proceed as follows:

1. We need to know Aut $Q$. In general, there is no algorithm to calculate the automorphism group of a finite group, unless the group belongs to a certain category such as $P$-groups or more generally nilpotent groups [Schönert et al. 1996]. But for small $Q$ this might be done by hand.

2. To find the set $\operatorname{Epim}\left(\pi_{g}, Q\right)$, we consider all tuples $q_{1}, q_{2}, \ldots, q_{2 g} \in Q$ generating $Q$ and satisfying the only defining relation of $\pi_{g}$ :

$$
r_{0}=\left[q_{1}, q_{2}\right]\left[q_{3}, q_{4}\right] \ldots\left[q_{2 g-1}, q_{2 g}\right]=1 .
$$

Aut $Q$ acts on the set of all these tuples. Let us choose a set of orbit representatives for this action. This yields the set $\operatorname{Epim}\left(\pi_{g}, Q\right) /$ Aut $Q$.

3. For every $\varphi \in \operatorname{Epim}\left(\pi_{g}, Q\right)$, we determine $N_{\varphi}=$ $\operatorname{Ker} \varphi$ as the normal closure of a finite set of elements of $\pi_{g}$ as follows:

We construct a presentation for $Q$ on generators $q_{1}, q_{2}, \ldots, q_{2 g}$. Besides $r_{0}$, the only relation of $\pi_{g}$, this presentation of $Q$ will satisfy some more relations, such as $r_{1}, r_{2}, \ldots, r_{k}$. If we rewrite $r_{1}$, $r_{2}, \ldots, r_{k}$ as words in generators of $\pi_{g}$, we will obtain a set whose normal closure will be $N$.

4. $\mathcal{M}_{g} \simeq \mathrm{Out}^{+}\left(\pi_{g}\right)$ acts on the conjugacy classes of $\pi_{g}$. Through hand calculation we determine the action of $D_{\alpha_{1}}, D_{\beta_{1}}, \ldots, D_{\alpha_{g}}, D_{\beta_{g}}, D_{\delta}$ (see Figure 3 ) on the conjugacy classes of the generators of $\pi_{g}$ (see Figure 3 ). We choose a fixed presentation of $\pi_{g}$ as follows:

$$
\left\langle a_{1}, b_{1}, \ldots, a_{g}, b_{g}:\left[a_{1}, b_{1}\right]\left[a_{2}, b_{2}\right] \ldots\left[a_{g}, b_{g}\right]=1\right\rangle .
$$

In Figure 3, we have drawn $2 g$ loops, with the appropriate orientation, whose isotopy classes represent the $2 g$ generators of $\pi_{g}$ satisfying the only relation of $\pi_{g}$. Here, $[a, b]=a b a^{-1} b^{-1}$ is the commutator of $a$ and $b$, and we multiply curves from left to right; that is, the curve $a b$ is obtained by traversing at first curve $a$, then curve $b$.

In addition to $D_{\alpha_{1}}, D_{\beta_{1}}, \ldots, D_{\alpha_{g}}, D_{\beta_{g}}, D_{\delta}$, we also need to know the action of $D_{\eta}$ (see Figure 3). The reason is the following (see [Johnson 1983]). The Torelli subgroup $\mathcal{T}_{g}$ is generated by all the elements of the form

$$
D_{\delta} D_{\eta}^{-1}
$$

where $\delta$ and $\eta$ represent a bounding pair, i.e., a pair of disjoint simple closed curves representing the same nontrivial $\mathbb{Z}$-homology class. Johnson further defines the genus of a bounding pair to be the smaller of the genera of the two pieces of the surface cut by the two curves, and proves that $\mathcal{T}_{g}$ is normally generated by any genus 1 bounding pair. Our choice in Figure 3 is of genus 1. This result will be used to settle problem 2 . That is, in

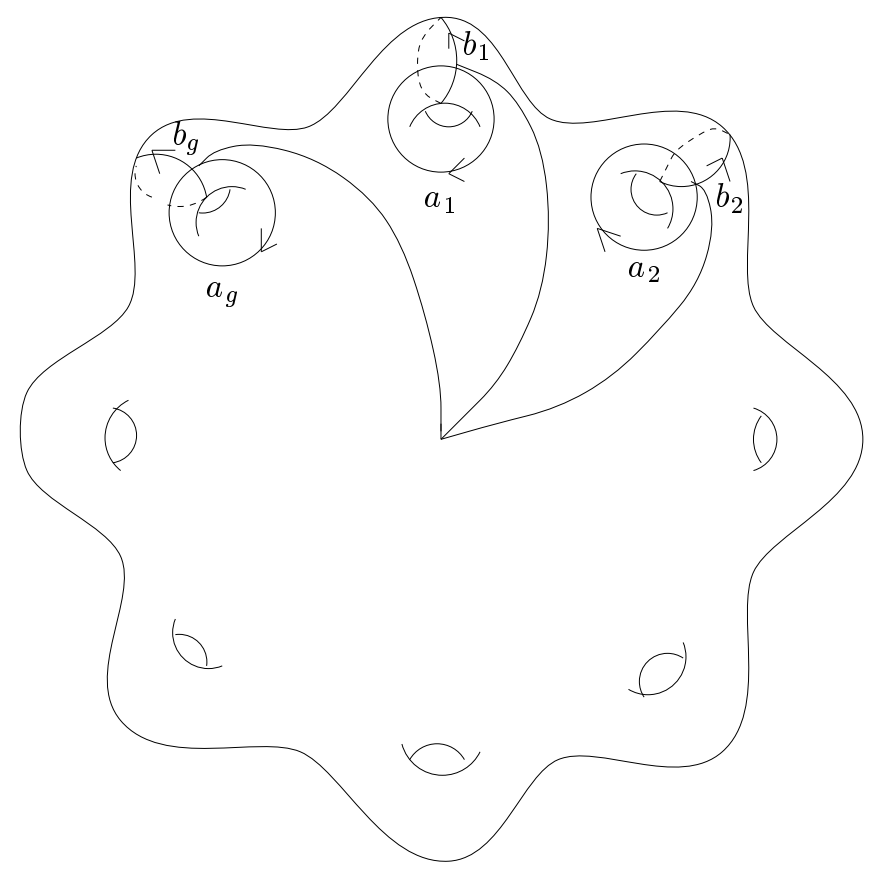

FIGURE 3. The generators of $\pi_{g}$. 
order to determine if a certain normal subgroup of $\mathcal{M}_{g}$ such as $\Sigma_{g, Q}$ contains $\mathcal{T}_{g}$ or not, all we have to do is determine whether

$$
\Phi_{g, Q}\left(D_{\delta} D_{\eta}^{-1}\right)
$$

is the trivial permutation, which can easily be done.

We will now list the action of the Dehn twists on the generators of $\pi_{g}$. Every Dehn twist changes only some of the generators. So we will not list those generators that remain invariant. We will need some new elements of $\pi_{g}$, defined as follows:

$$
\begin{array}{rlrl}
\alpha_{1} & =b_{1}^{-1}, & \\
\alpha_{i} & =a_{i} b_{i}^{-1} a_{i}^{-1} b_{i-1}, & & \text { for } i=2, \ldots, g, \\
\beta_{i} & =a_{i}, & & \text { for } i=1, \ldots, g, \\
\delta & =b_{2}, & \\
\eta & =\alpha_{3}^{-1} b_{3}^{-1} . &
\end{array}
$$

See Figure 4.

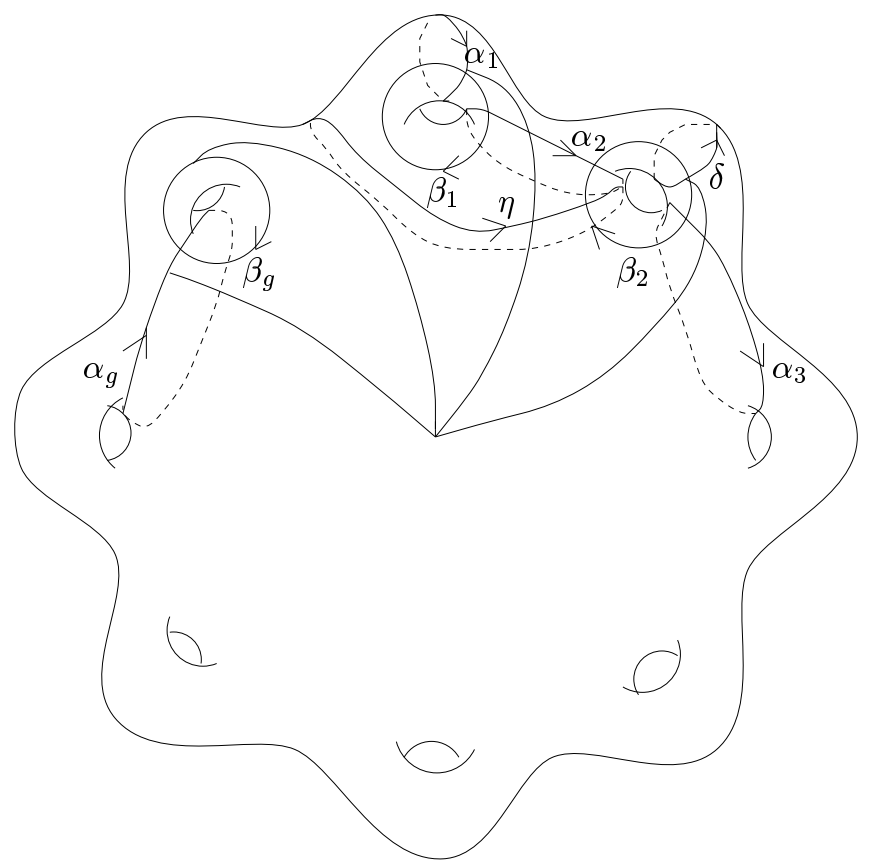

FIGURE 4. The action of Dehn twists.

These elements of $\pi_{g}$ are denoted by the same letters as the simple closed curves used in the presentation of $\mathcal{M}_{g}$ because they all happen to be simple closed curves representing the same isotopy classes. For the right Dehn twists, we get:

$$
\begin{aligned}
& D_{\alpha_{1}}: a_{1} \mapsto a_{1} \alpha_{1}, \\
& D_{\alpha_{i}}: a_{i-1} \mapsto a_{i-1} \alpha_{i}^{-1} \text {, } \\
& b_{i-1} \mapsto \alpha_{i} b_{i-1} \alpha_{i}^{-1} \text { for } i=2, \ldots, g \text {, } \\
& a_{i} \mapsto \alpha_{i} a_{i}, \\
& D_{\beta_{i}}: b_{i} \quad \mapsto b_{i} \beta_{i} \quad \text { for } i=1, \ldots, g, \\
& D_{\delta}: a_{2} \mapsto a_{2} \delta^{-1}, \\
& D_{\eta}: a_{2} \mapsto a_{2} \eta, \\
& b_{2} \mapsto \eta^{-1} b_{2} \eta \\
& a_{3} \mapsto \eta^{-1} a_{3} \eta \\
& b_{3} \mapsto \eta^{-1} b_{3} \eta \text {. }
\end{aligned}
$$

We will also need the action of the left Dehn twists:

$$
\begin{aligned}
& D_{\alpha_{1}}^{-1}: a_{1} \mapsto a_{1} \alpha_{1}^{-1}, \\
& D_{\alpha_{i}}^{-1}: a_{i-1} \mapsto a_{i-1} \alpha_{i}, \\
& b_{i-1} \mapsto \alpha_{i}^{-1} b_{i-1} \alpha_{i} \quad \text { for } i=2, \ldots, g, \\
& a_{i} \mapsto \alpha_{i}^{-1} a_{i}, \\
& D_{\beta_{i}}^{-1}: b_{i} \quad \mapsto b_{i} \beta_{i}^{-1} \quad \text { for } i=1, \ldots, g \text {, } \\
& D_{\delta}^{-1}: a_{2} \mapsto a_{2} \delta \text {, } \\
& D_{\eta}^{-1}: a_{2} \quad \mapsto a_{2} \eta^{-1}, \\
& b_{2} \mapsto \eta b_{2} \eta^{-1} \\
& a_{3} \mapsto \eta a_{3} \eta^{-1}, \\
& b_{3} \mapsto \eta b_{3} \eta^{-1} \text {. }
\end{aligned}
$$

5. Let $w=\omega\left(a_{1}, b_{1}, \ldots, a_{g}, b_{g}\right)$ be some word in the generators of $\pi_{g}$, and let $D_{\alpha}$ be some Dehn twist. Define

$D_{\alpha}(w)=\omega\left(D_{\alpha}\left(a_{1}\right), D_{\alpha}\left(b_{1}\right), \ldots, D_{\alpha}\left(a_{g}\right), D_{\alpha}\left(b_{g}\right)\right)$.

Now if $N$ is the normal closure of $n_{1}, n_{2}, \ldots, n_{l}$, where $n_{i}$ are some words in $a_{1}, b_{1}, \ldots, a_{g}, b_{g}$, then $D_{\alpha}(N)$ will be a normal subgroup normally generated by $D_{\alpha}\left(n_{1}\right), \ldots, D_{\alpha}\left(n_{l}\right)$. Now let $\varphi$ be an element of $\operatorname{Epim}\left(\pi_{g}, Q\right)$ and $N=N_{\varphi}$ be the corresponding kernel of $\varphi$. If we apply $D_{\alpha}$ to $N$, we will obtain another $N_{\varphi^{\prime}}$ for some other $\varphi^{\prime} \in$ $\operatorname{Epim}\left(\pi_{g}, Q\right)$. Since we know all $\varphi$ 's together with their kernels, we can find the appropriate normal subgroup that $N$ gets mapped to under the action of $D_{\alpha}$. In this way, we construct the homomorphism

$$
\Phi_{g, Q}: \mathcal{M}_{g} \rightarrow S_{k}
$$

In the next example we choose $Q$ to be the smallest nonabelian finite group $S_{3}$. First we need some facts from classical group theory. 
Definition 4.1. Let $G$ be a group and $H$ a permutation group on a finite set $\Omega$ with $|\Omega|=n$. The wreath product $G$ ? $H$ of $G$ with $H$ is defined to be the set

$$
G \nmid H=\left\{\left(g_{1}, g_{2}, \ldots, g_{n}, h\right): g_{i} \in G, h \in H\right\}
$$

with the group multiplication

$$
\begin{aligned}
\left(g_{1}, g_{2}, \ldots, g_{n}, h\right) & \left(g_{1}^{\prime}, g_{2}^{\prime}, \ldots, g_{n}^{\prime}, h^{\prime}\right) \\
& =\left(g_{1} g_{h(1)}^{\prime}, g_{2} g_{h(2)}^{\prime}, \ldots, g_{n} g_{h(n)}^{\prime}, h h^{\prime}\right) .
\end{aligned}
$$

Theorem 4.2. Let $G$ and $H$ be defined as in definition 4.1. Then $G\{H$ has a normal subgroup isomorphic to the direct product of $n$ copies of $G$ and is isomorphic to a semidirect product of this normal subgroup by $H$. Hence, the order of $G$ ? $H$ will be

$$
|G \curlywedge H|=|G|^{n}|H| \text {. }
$$

See [Huppert 1967] page 95 for a proof.

Definition 4.3. A permutation group $G$ on the set $\Omega$ is imprimitive if there exists a proper subset $\Delta \subset \Omega$ such that for all $g \in G$ either $g \Delta=\Delta$ or $g \Delta \cap \Delta=\varnothing$. $\Delta$ is called an imprimitivity region of $G$. A transitive permutation group that is not imprimitive is called primitive.

Theorem 4.4. Let $G$ be an imprimitive permutation group on $\Omega$ with imprimitivity region $\Delta$, and let $H=$ $\{g \in G: g \Delta=\Delta\}$ be the stabilizer of $\Delta$. Let further $R$ be a set of coset representatives of $G / H$.

(a) $\Omega=\bigcup_{r \in R} r \Delta$.

(b) If $|\Omega|=n$ is finite, then $|\Omega|=|\Delta|[G: H]$. Let $|\Delta|=k$ and $[G: H]=p$. Then $G$ is isomorphic to a subgroup of the wreath product of $S_{k} \prec S_{p}$.

\section{(c) $H$ acts transitively on $\Delta$.}

The proof can be found in [Huppert 1967, p. 146].

Theorem 4.5. Let $G=\left\langle g_{1}, g_{2}, \ldots, g_{m}: r_{1}, r_{2}, \ldots, r_{k}\right\rangle$ be a finitely presented group and

$$
\varphi: G \rightarrow S_{n}
$$

be a transitive permutation representation of $G$ on $n$ elements. Let us denote the image of $\varphi$ by $H$. Then we can construct a presentation for $H$ on $h_{1}=$ $\varphi\left(g_{1}\right), \ldots, h_{m}=\varphi\left(g_{n}\right)$ as follows

$$
H=\left\langle h_{1}, h_{2}, \ldots, h_{m}: r_{1}^{\prime}, r_{2}^{\prime}, \ldots, r_{k^{\prime}}^{\prime}\right\rangle
$$

(see Section 7), and $N=\operatorname{Ker} \varphi$ is normally generated by $r_{1}^{\prime}, r_{2}^{\prime}, \ldots, r_{k^{\prime}}^{\prime}$. (Note that $r_{1}^{\prime}, \ldots, r_{k^{\prime}}^{\prime}$ are the same words used in the presentation of $H$ but written in the generators of $G$.)
Let further $\left\{s_{1}, s_{2}, \ldots, s_{t}\right\}$ be a set of representatives of the preimages of all elements of $H$ under $\varphi$. Then a generating set for $N$ as a subgroup will be

$$
N=\left\langle s_{i}^{-1} r_{j}^{\prime} s_{i}: i=1, \ldots, t, j=1, \ldots, k^{\prime}\right\rangle .
$$

If $U$ is a subgroup of $H$ (such as the stabilizer of one point), generated by $u_{1}, \ldots, u_{l}$, and $w_{1}, \ldots, w_{l}$ are their preimage representatives in $G$, then $\varphi^{-1}(U)$ is generated as

$$
\left\langle w_{1}, \ldots, w_{l}, s_{i}^{-1} r_{j}^{\prime} s_{i}: i=1, \ldots, t, j=1, \ldots, k\right\rangle .
$$

The proof is trivial.

Now we are ready to look at some examples.

Example 4.6. $Q=S_{3}$.

Aut $S_{3}$ is isomorphic to $S_{3}$. The set

$$
\operatorname{Epim}\left(\pi_{g}, Q\right) / \text { Aut } S_{3}
$$

contains exactly 2520 elements; that is, there are exactly 2520 normal subgroups $N \unlhd \pi_{1}\left(\mathcal{S}_{3}\right)$ with $\pi_{1}\left(\mathcal{S}_{3}\right) / N \simeq S_{3}$. As a result, we obtain the homomorphism

$$
\Phi_{3, S_{3}}: \mathcal{M}_{3} \rightarrow S_{2520}
$$

$S_{3}$ is solvable with $S_{3}^{\prime} \simeq \mathbb{Z}_{3}$. As mentioned earlier in example 3.4 , there are exactly 63 subgroups $M_{1}, M_{2}, \ldots, M_{63}$ in $\pi_{1}\left(\mathcal{S}_{3}\right)$ with $\mathbb{Z}_{2}$-quotient. Each of these normal subgroups $M_{i}$ turns out to contain $N_{i, 1}, N_{i, 2}, \ldots, N_{i, 40}$ normal subgroups with $\mathbb{Z}_{3}$ as a quotient such that each $N_{i, j}$ is also normal in $\pi_{1}\left(\mathcal{S}_{3}\right)$ and $\pi_{1}\left(\mathcal{S}_{3}\right) / N_{i, j} \simeq S_{3}$. Let's denote the set of these subgroups by $\mathcal{N}_{S_{3}}=\left\{N_{i, j}\right\}$. In this way, we obtain a partitioning of these 2520 normal subgroups into 63 blocks, $\Delta_{1}, \ldots, \Delta_{63}$, each containing 40 normal subgroups. The action of $\mathcal{M}_{3}$ on $\mathcal{N}_{S_{3}}$ is transitive but not primitive. The imprimitivity regions are exactly the blocks $\Delta_{1}, \ldots, \Delta_{63}$. The permutation of the $\Delta_{i}^{\prime} s$ is determined by the action of $\mathcal{M}_{3}$ on the normal subgroups $M_{1}, M_{2}, \ldots, M_{63}$, which gives us a faithful transitive permutation representation of $S P_{6}\left(\mathbb{Z}_{2}\right)$ on 63 elements, as we saw in example 3.4. The stabilizer of one of the $\Delta_{i}^{\prime} s$, such as $\Delta_{1}$, for instance, under the action of $\mathcal{M}_{3}$ acts transitively on $\Delta_{1}$ (see Theorem 4.4). Therefore, the restriction of this action on $\Delta_{1}$ will be a transitive permutation representation on 40 elements. Let us denote the image of this representation by $U$. We can also compute a set of generators for $U$. Table 3 shows six permutations that generate $U$. 


$$
\begin{aligned}
& (5,23,32)(6,24,34)(7,25,33)(8,26,38)(9,27,40)(10,28,39)(11,29,35)(12,30,37)(13,31,36) \\
& (14,32,23)(15,33,24)(16,34,25)(17,35,26)(18,36,27)(19,37,28)(20,38,29)(21,39,30)(22,40,31) \\
& (2,3,4)(8,9,10)(11,13,12)(17,18,19)(20,22,21)(26,27,28)(29,31,30)(35,36,37)(38,40,39) \\
& (1,2,4)(6,11,10)(7,8,12)(15,20,19)(16,17,21)(24,29,28)(25,26,30)(33,38,37)(34,35,39) \\
& (2,3,4)(8,9,10)(11,13,12)(17,18,19)(20,22,21)(26,27,28)(29,31,30)(35,36,37)(38,40,39) \\
& (2,22,19)(3,21,17)(4,20,18)(5,25,34)(6,23,33)(7,24,32)(11,37,31)(12,36,29)(13,35,30)
\end{aligned}
$$

TABLE 3. Six permutations that generate $U$.

$U$ turns out to be a simple group of order 25920 . The only simple group of this order is $\mathrm{PSp}_{4}\left(\mathbb{Z}_{3}\right)$, (see [Conway et al. 1985]). Thus, we proved that

$$
\operatorname{Im}\left(\Phi_{3, S_{3}}\right) \simeq \operatorname{PSp}_{4}\left(\mathbb{Z}_{3}\right) \curlywedge \operatorname{Sp}_{6}\left(\mathbb{Z}_{2}\right)
$$

and the order of this image is

$$
(25920)^{63} \times 1451520 \approx 10^{284} .
$$

It's needless to mention that computing a presentation for $\Sigma=\Sigma_{3, S_{3}}$ is a hopeless task, but we are not actually interested in computing this presentation, but rather knowing if $H^{1}(\Sigma)$ is finite or not. We are going to look at $H=\Phi_{3, S_{3}}^{-1}\left(\operatorname{Stab}_{\Phi_{3, S_{3}}\left(\mathcal{M}_{3}\right)}\left(N_{1,1}\right)\right)$. As mentioned above, the image of $\mathcal{M}_{3}$ under $\Phi_{3, S_{3}}$ is transitive. So the stabilizer of one of the $\left\{N_{i, j}\right\}$, such as $N_{1,1}$, will be a subgroup of index 2520 , and its preimage $H$ will be a subgroup of the same index in $\mathcal{M}_{3}$. What seems to be realistic to handle is computing a presentation for $H$. On the other hand, $\Sigma_{3, S_{3}}=$ Core $_{\mathcal{M}_{3}}(H)=\bigcap_{g \in \mathcal{M}_{3}} g^{-1} H g$, meaning that $\mathcal{T}_{3} \leq \Sigma_{3, S_{3}}$ if and only if $\mathcal{T}_{3} \leq H$.

\section{COMPUTING A PRESENTATION FOR H}

Even 2520 is a huge index, and our computer won't be able to compute a presentation for $H$. Therefore, we will break down the calculations in two steps. At first, we look at $K=\Phi_{3, \mathbb{Z}_{2}}^{-1}\left(\operatorname{Stab}_{\Phi_{3, \mathbb{Z}_{2}}\left(\mathcal{M}_{3}\right)}\left(M_{1}\right)\right)$, which will be of index 63 in $\mathcal{M}_{3}$. Since $K$ fixes $M_{1}$, it acts on $\left\{N_{1,1}, \ldots, N_{1,40}\right\}$. Therefore, $\Phi_{3, S_{3}}$ can be restricted to $K$ and we will get a homomorphism (that we denote by the same letter)

$$
\Phi_{3, S_{3}}: K \rightarrow S_{40} .
$$

In the previous example, we denoted the image of $K$ by $U$. Now we look at $H=\Phi_{3, S_{3}}^{-1}\left(\operatorname{Stab}_{\Phi_{3, S_{3}}(K)}\left(N_{1,1}\right)\right)$, which will be a subgroup of index 40 in $K$, and a subgroup of index $40 \times 63=2520$ in $\mathcal{M}_{3}$. Using Theorem 4.5 , we can find a generating set for $K$. Then we construct a presentation for $K$ on this generating set; see Section 7. The advantage of this specific representation of $K$ is that every element of $K$ (written as a word in its generators) can be directly rewritten as a word in the generators of $\mathcal{M}_{3}$. This means that every subgroup of $K$ can be directly realized as a subgroup of $\mathcal{N}_{3}$, and any homomorphism from $\mathcal{M}_{3}$ onto any permutation group can be restricted on $K$ and easily evaluated. We can also find a generating set (using again Theorem 4.5) for $H$ as a subgroup of $K$ together with a presentation. Using the presentation, we can evaluate its first homology group, and taking advantage of the special presentation of $K$, we can realize $H$ as a subgroup of $\mathcal{M}_{3}$. The most time consuming part of the calculations involves the computation of a presentation for $H$. Here, it would be reasonable to check, at first, if $H$ contains the Torelli subgroup or not. The smallest generating set we can find for $K$ consists of the 8 elements

$$
a_{1}, b_{1}, a_{2}^{-2}, b_{2}, a_{3}, b_{3}, d^{-2}, a_{2} b_{2} d b_{2}^{-1} a_{2}^{-1} .
$$

Then, we calculate a presentation for $K$, and apply the Tietze transformations to simplify it. The simplest presentation we get has 8 generators and 242 relations of total length $66790 . H$ is generated by the 9 elements $a_{1}, b_{1}, a_{2}^{-2}, b_{2}, b_{3}, d^{-2}, a_{2} b_{2} d b_{2}^{-1} a_{2}^{-1}$, $a_{3} b_{2}^{-1} a_{3}, a_{3} b_{3} b_{2}^{-1} a_{3}^{-1}$, and does not contain the Torelli subgroup. $H$ has a presentation on 94 generators and 9401 relations of total length 2120026. At the end, we determine the commutator factor group of $H$. The nontrivial invariant divisors are 2,6 , and 12. Hence

$$
H_{1}(H) \simeq \mathbb{Z}_{2} \oplus \mathbb{Z}_{6} \oplus \mathbb{Z}_{12} .
$$

Although $H$ is a subgroup that does not contain the Torelli subgroup, its first cohomology group is zero.

The next attempt will be undertaken using the quaternion group $Q_{8}$ instead of $S_{3}$.

Example 5.1. $Q=Q_{8}$. 
$Q_{8}$ is a solvable group with $Q_{8}^{\prime} \simeq \mathbb{Z}_{2}$ and $Q_{8} / Q_{8}^{\prime} \simeq$ $\mathbb{Z}_{2} \oplus \mathbb{Z}_{2}$. We have exactly

$$
\frac{\left(2^{6}-1\right)\left(2^{6}-2\right)}{\left(2^{2}-1\right)\left(2^{2}-2\right)}=651
$$

subspaces of codimension two in $\left(\mathbb{Z}_{2}\right)^{6}$, so there are exactly 651 normal subgroups $M_{1}, \ldots, M_{651}$ in $\pi_{1}\left(\mathcal{S}_{3}\right)$ with $\mathbb{Z}_{2} \oplus \mathbb{Z}_{2}$ as quotient. The action of $\mathcal{M}_{3}$ on these subspaces won't be transitive. There are exactly two types of subspaces of codimension two, and the action of $\mathcal{M}_{3}$ will be transitive on each of these families. One of them is called isotropic, on which the symplectic form vanishes on its symplectic orthogonal complement, and the other one is called hyperbolic, on which the symplectic form is nondegenerate on its symplectic orthogonal complement (see [Huppert 1967]). In our situation, we will be only interested in the hyperbolic ones and there will be 315 of those. Each of these hyperbolic subgroups $M_{i}$ contains exactly 16 normal subgroups $\left\{N_{i, 1}, \ldots, N_{i, 16}\right\}$ such that their quotient in $\pi_{1}\left(\mathcal{S}_{3}\right)$ is isomorphic to $Q_{8}$. In this way, we obtain $315 \times 16=5040$ normal subgroups of quotient $Q_{8}$. The homomorphism

$$
\Phi_{3, Q_{8}}: \mathcal{M}_{3} \rightarrow S_{5040}
$$

will have an image isomorphic to $U$ \, where $L$ is a transitive permutation representation of $\operatorname{Sp}_{6}\left(\mathbb{Z}_{2}\right)$ on 315 elements and $U$ is a solvable permutation group of order 9216 on 16 elements. The order of this image will be

$$
9216^{315} \times 1451520 \approx 10^{1255} .
$$

Again, we look at

$$
K=\Phi_{3, \mathbb{Z}_{2} \oplus \mathbb{Z}_{2}}^{-1}\left(\operatorname{Stab}_{\Phi_{3, \mathbb{Z}_{2} \oplus \mathbb{Z}_{2}}\left(\mathcal{M}_{3}\right)}\left(M_{1}\right)\right)
$$

and at its subgroup $H=\Phi_{3, Q_{8}}^{-1}\left(\operatorname{Stab}_{\Phi_{3, Q_{8}}(K)}\left(N_{1,1}\right)\right)$. $K$ is a subgroup of index 315 containing the Torelli subgroup, and $H$ is a subgroup of index 5040 that does not to contain the latter group. $K$ is generated by the 10 elements $a_{1}, b_{1}, a_{2}^{-2}, b_{2}, a_{3}^{-2}, b_{3}, d^{-2}$, $a_{3} b_{3} b_{2}^{-1} a_{3}^{-1}, a_{2} b_{2} d b_{2}^{-1} a_{2}^{-1}$, and

$$
a_{2} b_{1} a_{1} b_{2} a_{2} b_{1} a_{3} b_{2} d a_{2}^{-1} b_{2}^{-1} a_{3}^{-1} b_{1}^{-1} a_{2}^{-1} b_{2}^{-1} a_{1}^{-1} b_{1}^{-1} a_{2}^{-1},
$$

and has a presentation with 10 generators and 1140 relations of total length 285918. $H$ is generated by the 13 elements $a_{1}, b_{1}, a_{2}^{4}, b_{2}, a_{3}^{4}, b_{3}, d^{4}, a_{3} b_{3} b_{2}^{-1} a_{3}^{-1}$, $a_{2} b_{2} d b_{2}^{-1} a_{2}^{-1}, a_{2}^{-2} b_{2} a_{2}^{2}, a_{2}^{-2} a_{3} b_{3} b_{2}^{-1} a_{3} a_{2}^{2}$,

$$
a_{2} b_{1} a_{1} b_{2} a_{2} b_{1} a_{3} b_{2} d a_{2}^{-1} b_{2}^{-1} a_{3}^{-1} b_{1}^{-1} a_{2}^{-1} b_{2}^{-1} a_{1}^{-1} b_{1}^{-1} a_{2}^{-1},
$$

and $a_{2}^{-2} b_{1}^{-2} a_{2}^{2}$, and has a presentation with 86 generators and 18105 relations of total length 3935640 . At the end we look at the commutator factor group of $H$. The nontrivial invariant divisors are $2,4,8$. Hence

$$
H_{1}(H) \simeq \mathbb{Z}_{2} \oplus \mathbb{Z}_{4} \oplus \mathbb{Z}_{8} .
$$

Therefore, $H^{1}(H)=0$.

We now list the results of our calculations for all the other choices for $Q$ that we have been able to handle. Although in each case the calculations are slightly different and need to be treated separately, we will not get into the details and will just state the results.

Example 5.2. $Q=D_{8}$.

There are 15120 normal subgroups of quotient $D_{8}$ (the dihedral group of order 8) in $\mathcal{M}_{3}$. The action of $\mathcal{N}_{3}$ on these 15120 subgroups is not transitive and has 2 orbits of equal length of 7560 elements. The stabilizer of one of the elements in the first orbit is a subgroup $H_{1}$ of index 7560 in $\mathcal{M}_{3}$ generated by 10 elements. A presentation on 126 generators and 27169 relations of total length 6069283 can be constructed for $H_{1}$. The nontrivial invariant divisors of $H_{1} / H_{1}^{\prime}$ are $2,2,2,4,4,4$. Hence

$$
H^{1}\left(H_{1}\right)=0 .
$$

As a matter of fact $H_{1}$ contains the Torelli subgroup. The stabilizer of one of the elements in the second orbit is a subgroup $\mathrm{H}_{2}$ of index 7560 in $\mathcal{M}_{3}$ generated by 10 elements. A presentation on 126 generators and 27168 relations of total length 6060990 can be constructed for $\mathrm{H}_{2}$. The nontrivial invariant divisors of $H_{2} / H_{2}^{\prime}$ are $2,2,4,8$. Hence

$$
H^{1}\left(H_{2}\right)=0 .
$$

It turns out that $H_{2}$ also contains the Torelli subgroup.

Example 5.3. $Q=D_{10}$.

There are 9828 normal subgroups of quotient $D_{10}$ in $\mathcal{M}_{3}$ falling into $\frac{9828}{63}=156$ blocks. The action of $\mathcal{M}_{3}$ on these 9828 subgroups is an imprimitive transitive group. The stabilizer of one of its element is a subgroup $H$ of index 9828 in $\mathcal{M}_{3}$ generated by 11 elements. A presentation on 259 generators and 
34795 relations of total length 5206667 can be constructed for $H$. The nontrivial invariant divisors of $H / H^{\prime}$ are $2,4,4$. Hence

$$
H^{1}(H)=0
$$

Here again $H$ contains the Torelli subgroup.

Example 5.4. $Q=D_{12}$.

There are 78120 normal subgroups of quotient $D_{12}$ (the dihedral group of order 12) in $\mathcal{M}_{3}$. The action of $\mathcal{N}_{3}$ on these subgroups is not transitive and falls into 4 orbits. We were not able to calculate a generating set much less a presentation for the stabilizer of any of these subgroups.

Example 5.5. $Q=D_{14}$.

There are 25200 subgroups of quotient $D_{14}$ in $\mathcal{M}_{3}$ falling into $\frac{25200}{63}=400$ blocks. The action of $\mathcal{M}_{3}$ on theses subgroups is an imprimitive transitive group. The stabilizer of one of its element is a subgroup $H$ of index 25200 in $\mathcal{M}_{3}$ generated by 11 elements. A presentation on 701 generators and 89308 relations of total length 12995940 can be constructed for $H$. However, we were not able to calculate the invariant divisors of $H / H^{\prime}$. But we could establish the fact that $H$ contains the Torelli subgroup, therefore

$$
H^{1}(H)=0 \text {. }
$$

Example 5.6. $Q=A_{4}$.

For $A_{4}$, the alternating group of order 12 , we were not even able to calculate a full list of normal subgroups in $\mathcal{M}_{3}$ having an $A_{4}$-quotient.

Example 5.7. $Q=T_{12}$.

In addition to $D_{12}$ and $A_{4}$ there is another nonabelian group $T_{12}$ of order 12, with presentation

$$
T_{12}=\left\langle t_{1}, t_{2}: t_{1}^{3}, t_{2}^{4}, t_{1} t_{2}^{-1} t_{1} t_{2}\right\rangle
$$

and permutation generators

$$
\begin{gathered}
(1,2,4)(3,6,5)(7,8,9)(10,12,11), \\
(1,3,7,10)(2,5,8,11)(4,6,9,12) .
\end{gathered}
$$

There are 80640 subgroups of quotient $T_{12}$ in $\mathcal{M}_{3}$ falling into $\frac{80640}{63}=1280$ blocks. The action of $\mathcal{M}_{3}$ on these group is an imprimitive transitive group. The stabilizer of one of its element is a subgroup $H$ of index 80640 in $\mathcal{M}_{3}$ generated by 24 elements. We were able to construct a presentation on 2336 generators and 287680 relations of total length 40604058 for $H$. Although we were not able to calculate the invariant divisors, we could establish that $H$ contains the Torelli subgroup, therefore

$$
H^{1}(H)=0 \text {. }
$$

The choices for $Q$, considered in the examples above, were the only cases we were able to handle for $g=3$. However, for $g=2$ we were able to construct more subgroups.

\section{MORE EXAMPLES FOR $\mathrm{g}=2$}

In the following we will list some more subgroups we have been able to construct for $\mathcal{M}_{2}$ using the method we described in this section.

Example 6.1. $Q=S_{3}$.

There are 60 normal subgroups of quotient $S_{3}$ in $\mathcal{M}_{2}$. The action of $\mathcal{M}_{2}$ on these 60 subgroups is a transitive permutation group. The stabilizer of one of these subgroups is a subgroup $H$ of index 60 in $\mathcal{M}_{2}$ generated by 7 elements. A presentation on 11 generators and 165 relations of total length 3496 can be constructed for $H$. The nontrivial invariant divisors of $H / H^{\prime}$ are $2,2,0,0$. Hence

$$
H^{1}(H)=\mathbb{Z} \oplus \mathbb{Z}
$$

Example 6.2. $Q=D_{8}$.

There are 180 normal subgroups of quotient $D_{8}$ in $\mathcal{M}_{2}$. The action of $\mathcal{M}_{2}$ on these 180 subgroups has 2 orbits of equal length 90 . The stabilizer of one of these subgroups in the first orbit is a subgroup $H_{1}$ of index 90 in $\mathcal{M}_{2}$ generated by 8 elements. A presentation on 35 generators and 391 relations of total length 6675 can be constructed for $H_{1}$. The nontrivial invariant divisors of $H_{1} / H_{1}^{\prime}$ are 2, 2, 2, 0 , 0,0 . Hence

$$
H^{1}\left(H_{1}\right)=\mathbb{Z} \oplus \mathbb{Z} \oplus \mathbb{Z}
$$

The stabilizer of one of the subgroups in the second orbit is a subgroup $\mathrm{H}_{2}$ of index 90 in $\mathcal{M}_{2}$ generated by 7 elements. A presentation on 36 generators and 393 relations of total length 6682 can be constructed for $\mathrm{H}_{2}$. The nontrivial invariant divisors of $\mathrm{H}_{2} / \mathrm{H}_{2}^{\prime}$ are $2,2,8,0,0$. Hence

$$
H^{1}\left(H_{2}\right)=\mathbb{Z} \oplus \mathbb{Z}
$$

Example 6.3. $Q=Q_{8}$. 
There are 60 normal subgroups of quotient $Q_{8}$ in $\mathcal{M}_{2}$. The action of $\mathcal{M}_{2}$ on these 60 subgroups is a transitive permutation group. The stabilizer of one of these subgroups is a subgroup $H$ of index 60 in $\mathcal{M}_{2}$ generated by 11 elements. A presentation on 24 generators and 263 relations of total length 4562 can be constructed for $H$. The nontrivial invariant divisors of $H / H^{\prime}$ are $2,2,4,0$. Hence

$$
H^{1}(H)=\mathbb{Z} \text {. }
$$

Example 6.4. $Q=D_{10}$.

There are 90 normal subgroups of quotient $D_{10}$ in $\mathcal{M}_{2}$. The action of $\mathcal{M}_{2}$ on these 90 subgroups is a transitive permutation group. The stabilizer of one of these subgroups is a subgroup $H$ of index 90 in $\mathcal{M}_{2}$ generated by 7 elements. A presentation on 15 generators and 245 relations of total length 5063 can be constructed for $H$. The nontrivial invariant divisors of $H / H^{\prime}$ are $2,2,4,0,0$. Hence

$$
H^{1}(H)=\mathbb{Z} \oplus \mathbb{Z} .
$$

Example 6.5. $Q=T_{12}$.

There are 480 normal subgroups of quotient $T_{12}$ in $\mathcal{M}_{2}$. The action of $\mathcal{M}_{2}$ on these 480 subgroups is a transitive permutation group. The stabilizer of one of the these subgroups $H$ is a subgroup of index 480 in $\mathcal{M}_{2}$ generated by 11 elements. A presentation on 68 generators and 1370 relations of total length 23395 can be constructed for $H$. The nontrivial invariant divisors of $H / H^{\prime}$ are 2, 2, 2, 0, 0, 0. Hence

$$
H^{1}(H)=\mathbb{Z} \oplus \mathbb{Z} \oplus \mathbb{Z}
$$

\section{FINAL REMARKS}

As mentioned in the introduction, almost all of the calculations have been performed in the programming language GAP. The programs were written by the author, except for the following two cases, where some new programs, that at the time of writing this paper were not yet implemented in GAP's library, were needed in:

1. calculating a presentation for a finite permutation group (see Theorem 4.5), and

2. producing a presentation on a given set of generators (see Section 5).
My special thanks goes to T. Brauer from RWTHAachen, not only for providing the programs in advance, but also for his valuable help and advice.

The first machine we have used for the purpose of our calculation was an Sun Ultrasparc 1 Model 170 (170 MGHz, and 128 Mbytes of RAM). The calculations for the groups $S_{3}, Q_{8}$ and $D_{8}$ were carried out using this machine. The three major parts of the calculations involve

1. calculating $\operatorname{Epim}\left(\pi_{g}, Q\right)$ (Section 4$)$,

2. evaluating $\Phi_{g, Q}$ (Section 4$)$,

3. producing the presentations for $H$ and $K$ (Section 5).

Part 3 is the most time consuming. The first two parts together take only about 20 percent of the total computing time. Here are the times needed for Examples 4.6, 5.1 and 5.2:
1. $Q=S_{3}, 52 \mathrm{~min}$,
2. $Q=Q_{8}, 283 \mathrm{~min}$,
3. $Q=D_{8}, 415 \mathrm{~min}$.

The other groups were handled with a faster machine: a $400 \mathrm{MGHz}$ Pentium II with 256 Mbyte of RAM.

Although we have been able to construct only a few number of subgroups inside $\mathcal{M}_{3}$, all of them have turned out to have a trivial first cohomology group. Unfortunately, the fact that almost all of them (except for $Q=S_{3}$ ) contain the Torelli subgroup makes it very difficult for us to guess whether $\mathcal{M}_{3}$ has the Kazhdan property. Considering that in our calculation $Q$ has always been a solvable group, the choice of a simple group for $Q$ would be an interesting case that deserves attention. Although the calculations for a simple $Q$ might shed some light on this problem, even the smallest choice $A_{5}$ (the alternating group on 5 elements) of order 60 turns out too big for the current methods.

\section{ELECTRONIC AVAILABILITY}

The GAP programs used in our calculations are about 2300 lines long and can be found at http:// www.math.msu.edu/ ferry. 


\section{REFERENCES}

[Bass et al. 1967] H. Bass, J. Milnor, and J.-P. Serre, "Solution of the congruence subgroup problem for $\mathrm{SL}_{n}$ $(n \geq 3)$ and $\operatorname{Sp}_{2 n}(n \geq 2)$ ", Publ. Math. Inst. Hautes Études Sci. 33 (1967), 59-137. Erratum in 44 (1974), 241-244.

[Birman and Hilden 1971] J. S. Birman and H. M. Hilden, "On the mapping class groups of closed surfaces as covering spaces", no. 66 (1971), 81-115.

[Conway et al. 1985] J. H. Conway, R. T. Curtis, S. P. Norton, R. A. Parker, and R. A. Wilson, Atlas of finite groups, Oxford University Press, Oxford, 1985.

[Dehn 1938] M. Dehn, "Die Gruppe der Abbildungsklassen", Acta Math. 69 (1938), 135-206.

[Gilman 1977] R. Gilman, "Finite quotients of the automorphism group of a free group", Canad. J. Math. 29:3 (1977), 541-551.

[Grossman 1974] E. K. Grossman, "On the residual finiteness of certain mapping class groups", J. London Math. Soc. (2) 9 (1974), 160-164.

[de la Harpe and Valette 1989] P. de la Harpe and A. Valette, La propriété $(T)$ de Kazhdan pour les groupes localement compacts, Astérisque 175, 1989. With an appendix by M. Burger.

[Humphreys 1980] J. E. Humphreys, Arithmetic groups, Lecture Notes in Math. 789, Springer, Berlin, 1980.

[Humphries 1979] S. P. Humphries, "Generators for the mapping class group", pp. 44-47 in Topology of low-dimensional manifolds (Chelwood Gate, Sussex, 1977), edited by R. Fenn, Lecture Notes in Math. 722, Springer, Berlin, 1979.

[Humphries 1992] S. P. Humphries, "Normal closures of powers of Dehn twists in mapping class groups", Glasgow Math. J. 34:3 (1992), 313-317.

[Huppert 1967] B. Huppert, Endliche Gruppen, I, Grundlehren der Math. Wissenschaften 134, Springer, Berlin, 1967.

[Ivanov 1984] N. V. Ivanov, "Algebraic properties of the Teichmüller modular group", Dokl. Akad. Nauk SSSR 275:4 (1984), 786-789. In Russian; translated in Soviet Math. Dokl 29:2.

[Ivanov 1986] N. V. Ivanov, "Algebraic properties of the Teichmüller modular group", no. 18 (1986).

[Ivanov 1987] N. V. Ivanov, "Complexes of curves and Teichmüller modular groups", Uspekhi Mat. Nauk
42:3(255) (1987), 49-91, 255. In Russian; translated in Russian Math. Surveys 42:3, 55-107.

[Ivanov 1994] N. V. Ivanov, "Ten problems on the mapping class groups", preprint, 1994.

[Johnson 1976] D. L. Johnson, Presentations of groups, London Math. Soc. Lecture Notes Series 22, Cambridge Univ. Press, Cambridge, 1976.

[Johnson 1980a] D. Johnson, "Conjugacy relations in subgroups of the mapping class group and a grouptheoretic description of the Rochlin invariant", Math. Ann. 249:3 (1980), 243-263.

[Johnson 1980b] D. L. Johnson, Topics in the theory of group presentations, London Math. Soc. Lecture Notes Series 42, Cambridge Univ. Press, Cambridge, 1980.

[Johnson 1983] D. Johnson, "A survey of the Torelli group", pp. 165-179 in Low-dimensional topology (San Francisco, 1981), edited by J. Samuel J. Lomonaco, Contemporary Math. 20, Amer. Math. Soc., Providence, 1983.

[Kazhdan 1967] D. A. Kazhdan, "Connection of the dual space of a group with the structure of its closed subgroups", Funkts. Anal. Prilozh. 1:1 (1967), 71-74. In Russian; translated in Funct. Anal. Appl. 1 (1967), 63-65.

[Lickorish 1964] W. B. R. Lickorish, "A finite set of generators for the homeotopy group of a 2-manifold", Proc. Cambridge Philos. Soc. 60 (1964), 769-778. Corrigendum in 62 (1966), 679-681.

[Lubotzky 1994] A. Lubotzky, Discrete groups, expanding graphs and invariant measures, Birkhäuser, Basel, 1994.

[Magnus et al. 1966] W. Magnus, A. Karrass, and D. Solitar, Combinatorial group theory: Presentations of groups in terms of generators and relations, Pure and Applied Math. 13, Interscience, New York, 1966.

[McCarthy 1996] J. D. McCarthy, "On the first cohomology group of cofinite subgroups in surface mapping class groups", Preprint, 1996.

[McCool 1975] J. McCool, "Some finitely presented subgroups of the automorphism group of a free group", J. Algebra 35 (1975), 205-213.

[Mennicke 1965] J. Mennicke, "Zur Theorie der Siegelschen Modulgruppe", Math. Ann. 159 (1965), 115129.

[Nielsen 1927] J. Nielsen, "Untersuchung zur Topologie der geschlossenen zweiseitigen Flächen I", Acta Math. 50 (1927), 189-358. 
[Nielsen 1929] J. Nielsen, "Untersuchung zur Topologie der geschlossenen zweiseitigen Flächen II", Acta Math. 53 (1929), 1-76.

[Nielsen 1931] J. Nielsen, "Untersuchung zur Topologie der geschlossenen zweiseitigen Flächen III", Acta Math. 58 (1931), 87-167.

[Powell 1978] J. Powell, "Two theorems on the mapping class group of a surface", Proc. Amer. Math. Soc. 68:3 (1978), 347-350.
[Schönert et al. 1996] M. Schönert et al., GAP: Groups, algorithms, and programming, 5th ed., Lehrstuhl D für Mathematik, RWTH Aachen, 1996. See http:// www-gap.dcs.st-and.ac.uk/ gap.

[Wajnryb 1983] B. Wajnryb, "A simple presentation for the mapping class group of an orientable surface", Israel J. Math. 45:2-3 (1983), 157-174. Errata by J. S. Birman and B. Wajnryb in 88:1-3 (1994), 425-427.

Feraydoun Taherkhani, Michigan State University, Department of Mathematics, Wells Hall, East Lansing, MI 48824, United States (ferry@math.msu.edu)

Received February 10, 1998; accepted in revised form May 25, 1999 